AperTO - Archivio Istituzionale Open Access dell'Università di Torino

\title{
Solid-Phase Synthesis of Asymmetric Cyanine Dyes
}

\section{This is a pre print version of the following article:}

Original Citation:

Availability:

This version is available http://hdl.handle.net/2318/1800493

since 2021-12-06T11:03:33Z

Published version:

DOI: $10.2174 / 1385272825666210628104642$

Terms of use:

Open Access

Anyone can freely access the full text of works made available as "Open Access". Works made available under a Creative Commons license can be used according to the terms and conditions of said license. Use of all other works requires consent of the right holder (author or publisher) if not exempted from copyright protection by the applicable law. 


\title{
MINI REVIEW ARTICLE
}

\section{Solid-Phase Synthesis of Asymmetric Cyanine Dyes}

\author{
Marco Giordano $^{\mathrm{a}}$, Giacomo Renno ${ }^{\mathrm{a}}$, Pierluigi Quagliotto ${ }^{\mathrm{a}}$, Claudia Barolo $^{\mathrm{a}}$, Giancarlo Cravotto ${ }^{\mathrm{b}}$, \\ Andrea Fin ${ }^{\mathrm{b}^{*}}$ and Guido Viscardi ${ }^{\mathrm{a}}$
}

${ }^{a}$ Department of Chemistry and NIS Interdepartmental Center and INSTM Reference Centre, University of Torino, Via Pietro Giuria 7 - 10125, Torino, Italy., ${ }^{b}$ Department of Drug Science and Technology, University of Torino, Via Pietro Giuria 9 - 10125, Torino, Italy.

\begin{abstract}
Cyanine dyes (CD) are a functional class of organic molecules used in several applications ranging from photography to bioimaging. CDs most well-known features resides on high molar extinction coefficients up to $10^{5} \mathrm{~L} \mathrm{~mol}^{-1} \mathrm{~cm}^{-1}$ and on the absorption spectra, ranging from 500 to 1000 $\mathrm{nm}$, which can be fine-tuned both by extending the length of the central methylene bridge or by modulating the terminal heterocycles. In the last decades, new synthetic methodologies, namely microwave-assisted and the solid-phase procedure, have been developed to overcome the limitation of the classical synthetic protocols. While microwave approach usually reduces the exposure time of the reagents and products to thermal degradation, the solid-phase methodology allows easier synthetic protocols which is translated in higher yields and simpler products purification. In the present review a comprehensive analysis of the solid-phase methods for the synthesis of asymmetrical CDs is discussed, with a critical evaluation of the difference among the currently available solid-state approaches.
\end{abstract}

Keywords: Cyanine Dyes, Asymmetric Dyes, Solid State synthesis, Resins, Solvent-free, User-friendly method

\section{INTRODUCTION}

Cyanine dyes $(\mathrm{CD})$ are a class of organic functional dyes characterized by a chemical structure in which two nitrogen atoms are linked through a single or multiple methine group to form a delocalized system containing an odd number of atoms (Figure 1). The overall structure is fully conjugated with the $\pi$ electrons delocalized along the whole molecular backbone while the positive charge is located on the nitrogen making the CDs depictable as a resonance hybrid of two structures [1-4]. By a structural point of view, the conjugated carbon bridge plays a key role on the photophysical properties of CDs leading to a bathochromic shift of around $100 \mathrm{~nm}$ upon each addition of a methylene unit (Figure 2) [4-5]. Cyanines can thus cover a large part of the visible spectra by simply tuning the length of the polymethine bridge. This peculiar features along with the remarkable molar extinction coefficients $\approx 1.5-3 \times 10^{5} \mathrm{~L} \mathrm{~mol}^{-1} \mathrm{~cm}^{-1}$ made CDs one of the most explored class of chromophores for various applications like photography, sensors, fluorescence imaging, data storage, nucleic acid labelling, medicine, dyessensitized solar cells [5-24]. CDs are generally named according to the length of the chain between the two nitrogen atoms.

*Address correspondence to this author at the Department of Drug Science and Technology, University of Torino, Via P. Giuria 9, Torino, Italy; Tel: +39-011-670-7887; E-mails: andrea.fin@unito.it
The dyes with one, three, five and seven methine units are called mono-, tri-, penta- and heptamethine respectively [4].
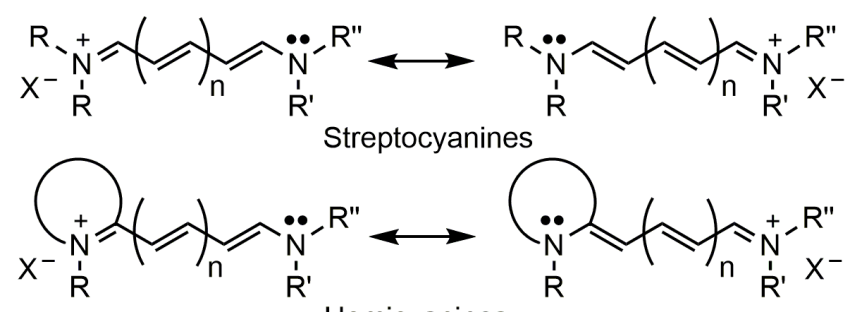
Hemicyanines



$\mathrm{n}=0,1,2,3 \quad$ Closed chain cyanines

Figure 1. Schematic representation of the different CDs as a function of the number of methine units and terminal groups.

However, an additional classification is commonly reported based on the chemical structure of the groups bearing the nitrogen atoms (Figure 1). Cyanines without any terminal heterocyclic groups are named streptocyanines, those with only one terminal heterocycle are hemicyanines and, those with two heterocycles at the chain edges are called closed chain cyanines [25-26]. 


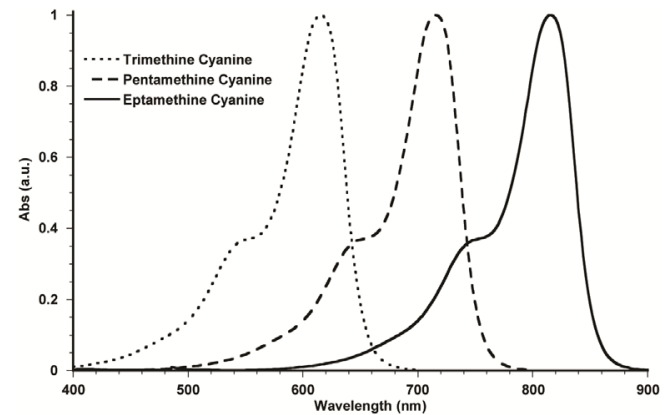

Figure 2. Bathochromic shift of the absorption spectra of symmetric CDs by extending the length of the methine bridge from tri-, to penta- and finally hepta- CDs.

In the latter family, a further subclassification is used by the nature of the heterocyclic structures such as indole, benzoindole, benzoxazole, benzothiazole, benzoselenazole, quinaldine and lepidine (Figure 3). The nature of the two terminal groups not only classifies the CDs but also affects the electronic behaviour of the overall dye. When the terminal moieties on the edges of the polymethine chain are identical, the dye has an even electron density distribution and these cyanines are called symmetrical. On the other hand, when the termini are different one to another the CDs are defined asymmetrical [27]. In both cases, for synthetic purposes, the heterocyclic precursors must carry a methyl group in $\alpha$ o $\gamma$ position to nitrogen atom whose quaternization increases methyl acidity. The common synthetic strategy to prepare symmetrical CDs, except for the monomethine cyanines, resides on the condensation of two equivalents of quaternary ammonium salts with the polymethine chain linker in a one-pot procedure $[4,28]$.
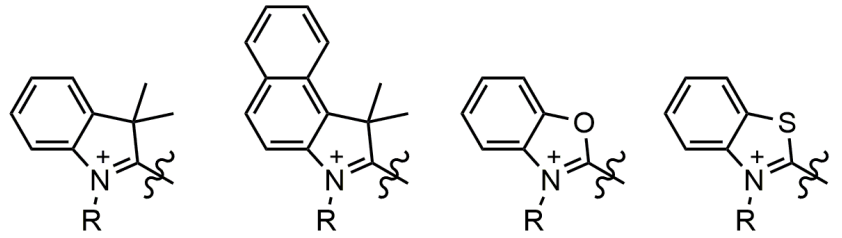

Indole

Benzoindole

Benzoxazole Benzothiazole



Quinaldine

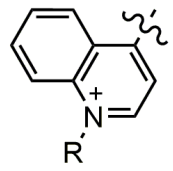

Lepidine
Benzoselenazole

Figure 3. Schematic representation of the heterocyclic moieties used to synthetize CDs.

The general reaction mechanism implies several steps starting with (i) a base (e.g. triethylamine, pyridine, acetate salts) promoted deprotonation at the $\alpha$ or $\gamma$ methyl group of the quaternary ammonium salt, leading to the formation of a nucleophilic methylene unit (Figure 4). The active methylene (ii) attacks the dianilide-based linker forming a new carboncarbon bond and (iii) promoting an electronic reorganization which releases a secondary amine as a leaving group. A final proton extraction (iv), by a base, leads to the formation of the hemicyanine with a full conjugated structure. The hemicyanine is then subjected to the same reaction pathway ( $\mathrm{v}$ and vi) with another equivalent of the quaternary salt to provide the final symmetrical dye.
According to the desired final CDs structure, various precursors can be used such as orthoesters for trimethine bridge, malondialdehydes and their dianilide-based derivatives for pentamethine scaffolds, glutaconaldehyde dianilide and Vilsmeier-Haack reagent for heptamethine dyes. This synthetic approach can also be applied to the synthesis of unsymmetrical dyes. Nevertheless, due to the presence of the concurrent formation of the symmetrical analogs, the asymmetrical dyes are generally formed in low yield and upon challenging purification. To overcome these limitations arising from the one-pot synthetic procedure, unsymmetrical CDs can be accessed by different methodology based on the polymethine bridge length [29].

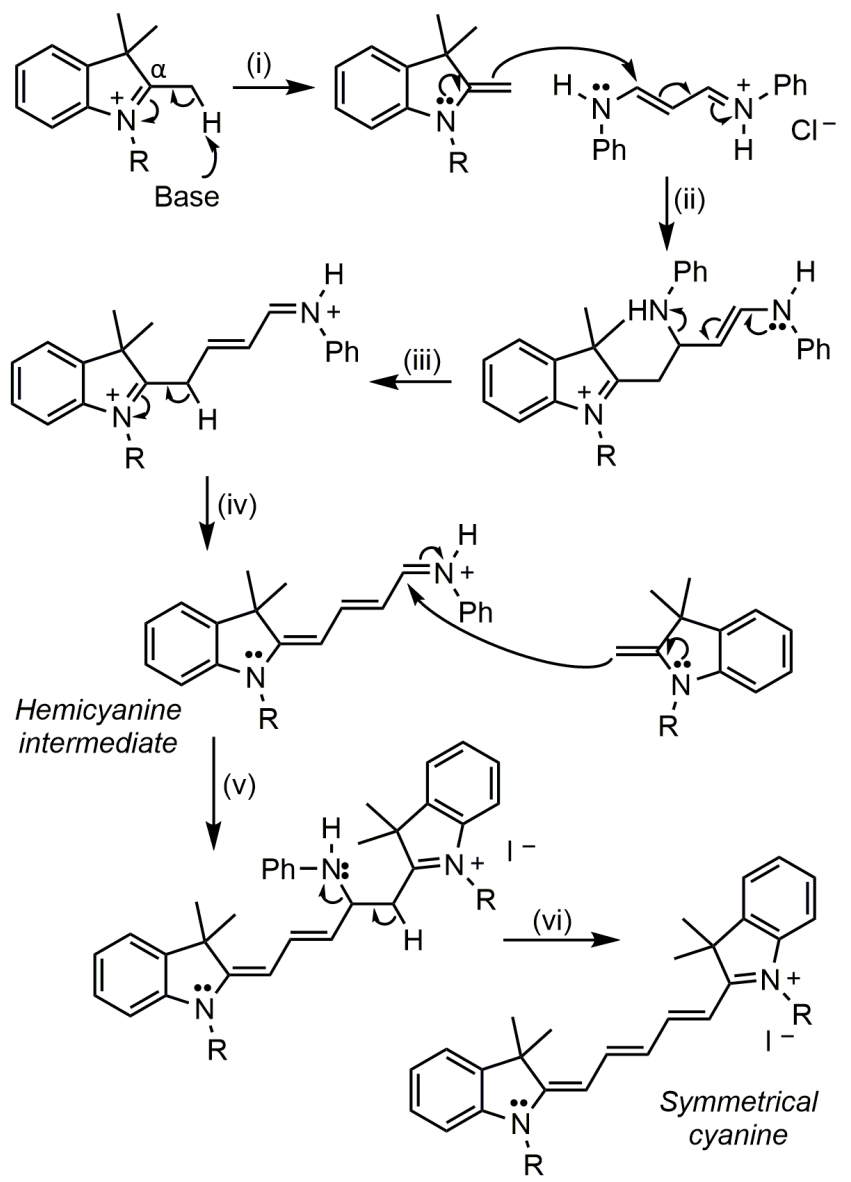

Figure 4. General reaction mechanism to form symmetrical CDs. (i) Deprotonation of the $\alpha$ methyl group of the quaternary ammonium salt; (ii) attack of the dianilide-based linker by the activated methylene; (iii) electronic reorganization and release of the secondary amine; (iv) formation of the hemicyanine scaffold; (v) attack of the hemicyanine by another activated quaternary salt; (vi) electronic reorganization and elimination of the secondary amine to yield the symmetrical cyanine.

The monomethine CDs can be easily obtained by a single step procedure based on the "thioether method" while tri-, penta- and heptamethine dyes can be accessed by multistep methods, namely the "aldehyde method" and the "hemicyanine method" (Figure 5a and 5b). The "aldehyde method" involves the use of a methine source and of a quaternary salt to yield an aldehyde derivative which reacts in a second step with a different quaternary salt obtaining the final unsymmetrical structure. The appropriate choice of the methine source addresses the length of the methine bridge. 


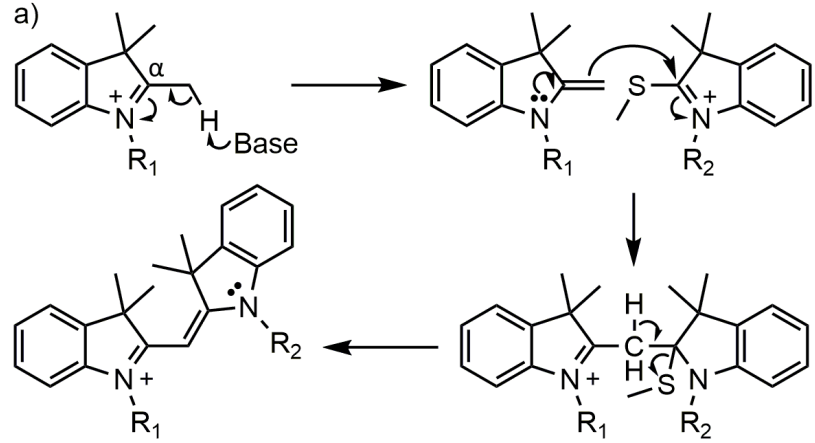

Asymmetrical monomethine cyanine

b)
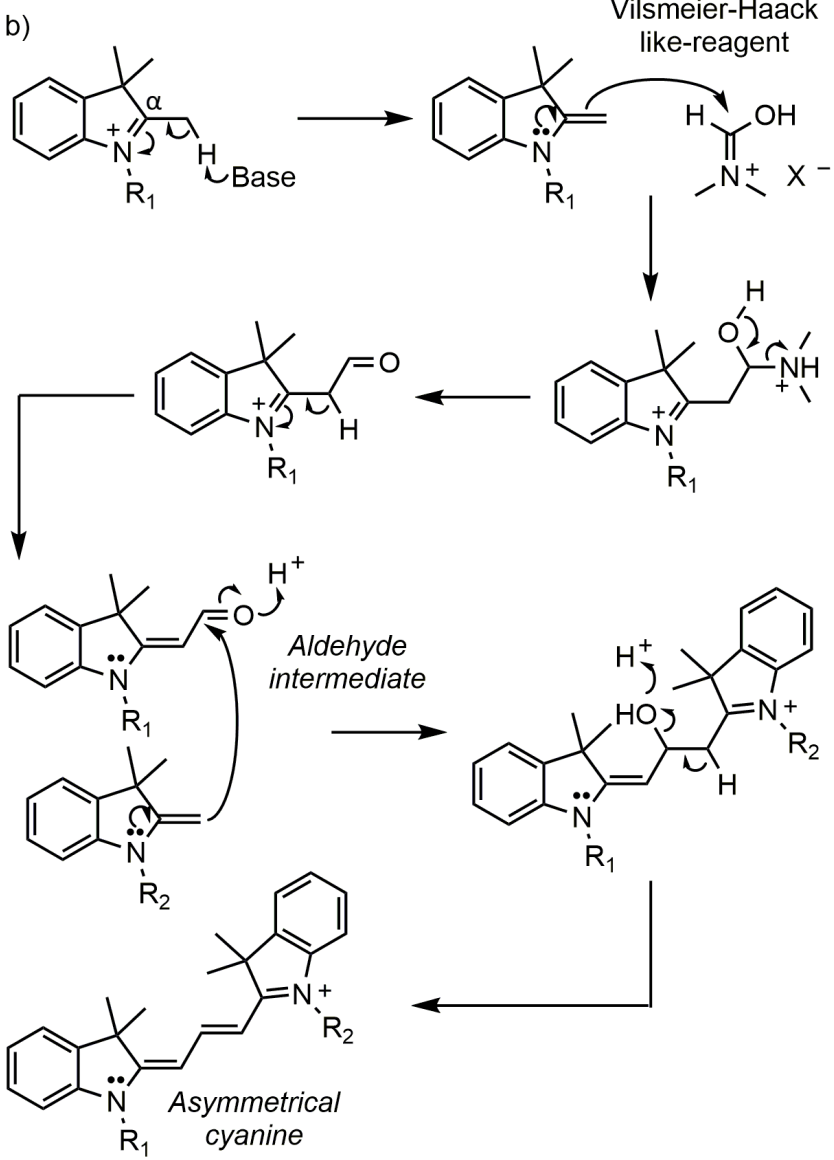

Figure 5. a) General mechanism of "thioether method". b) General mechanism of "aldehyde method".

$\mathrm{POCl}_{3}$ and $N, N$-dimethylformamide provide trimethine dyes, pentamethine CDs are prepared with tetraethoxypropane while 1-(dimethylamino)-5-formyl-1,3-butadiene is usually employed in the preparation of the heptamethines analogs. The "hemicyanine method" resembles the procedure described for the preparation of the symmetrical dyes but differs on the milder reaction conditions and ratio between the reagents. The first step involves the formation of the hemicyanine structure, while the second one provides the final asymmetrical cyanine by reaction with the nucleophilic methylene of another quaternary salt. Usually the hemicyanine intermediate requires to be activated, for example by $\mathrm{N}$-acylation to ensure an efficient reaction with the second quaternary salt. Nevertheless, during the first step, the formation of the undesired symmetrical dye cannot be completely avoided even by performing the coupling with an excess of polymethine linker. Although the abovementioned approaches ensure the synthesis of the desired asymmetrical CDs, it is worth noting that multistep procedures and non-trivial purifications are required [30].

Solid-state synthesis applying resin-based methodology or solvent-free procedures have been introduced in the last decades to overcome these limitations and providing CDs with higher yield and easier to be purified. In this short review, we report a comprehensive analysis of the nowadays available different solid-phase methods applied in the synthesis of asymmetrical cyanine dyes.

\section{SOLID-PHASE SYNTHESIS OF ASYMMETRICAL CYANINES}

Solid-phase organic synthesis and combinatorial chemistry are well-known approaches, involving the use of resins, for the synthesis of peptides and drugs [31, 32]. However, in the last years they have found a broader application in the synthesis of different type of dyes such as styryl dyes, BODIPYs, phthalocyanines and cyanines [33-42]. In particular, the use of solid-phase synthesis has become a powerful technique to cleanly synthesize asymmetrical CDs with high degree of purity and without tricky and expensive purification protocols. Two main methodologies have been developed based on the solid-phase approach, the "catchand-release" method and the "immobilized imidate" method. The former involves three distinct steps: (i) the synthesis of hemicyanine scaffold, (ii) the catch of the hemicyanine onto a resin, (iii) the final formation and later cleavage of the unsymmetrical cyanine dye from the resin (Figure 6) [38].



Figure 6. "Catch-and-release" solid-phase synthesis. (i) Synthesis of the hemicyanine by classical in solution methodology, (ii) immobilization of hemicyanine onto resin, (iii) formation and later cleavage of the unsymmetrical cyanine dye from the resin.

The immobilization of the hemicyanine onto the resin has the dual function of activating the hemicyanine and of facilitating the removal of the major byproducts. The hemicyanine intermediates are relatively unreactive at room temperature but can be activated by functionalizing the nitrogen with an electron-withdrawing group like by acetylation or sulfonylation $[43,44]$. In a similar manner, the reaction of the hemicyanine with a sulfonyl chloride polymer-bound resin not only activates the substrate but also allows the removal of the undesired byproducts, mainly the symmetrical $\mathrm{CD}$, by simple washing the resin with compatible solvents. Finally, the reaction of bound- 
hemicyanine with another heterocyclic carbon nucleophile leads the concurrent formation of the desired asymmetrical $\mathrm{CD}$ and the release from the resin. Nevertheless, it must be pointed out that the use of a substoichiometric amount of the second heterocyclic nucleophile is crucial in the cleavage step to minimize the contamination of the final product. The pure product can be easily obtained by the separation of resin by filtration. The "catch-and-release" methods allows an easier purification of the final dye in comparison to the classical synthesis in solution but still requires the synthesis and purification of the hemicyanine scaffold which, usually, is not so straightforward.
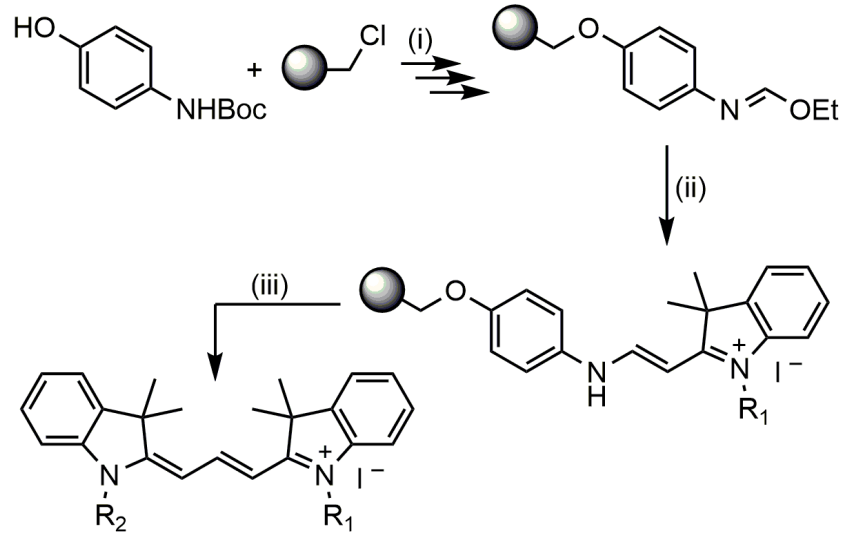

Figure 7. "Immobilized imidate" solid-phase synthesis. (i) Construction of resin-bounded imidate, (ii) Synthesis of immobilized hemicyanine, (iii) formation and later cleavage of the unsymmetrical cyanine dye from the resin.

The "immobilized imidate" method was developed to overcome the above-mentioned limitation of the "catch-andrelease" procedure. The approach is based on three distinct steps: (i) the construction of resin-bounded imidate, (ii) the synthesis of a polymer-bounded hemicyanine scaffold, (iii) the final formation and the later cleavage of the unsymmetrical cyanine dye from the resin (Figure 7) [39]. The "immobilized imidate" method allows an easier purification of hemicyanine due to the immobilization onto resin since the first synthetic step in comparison to the "catch-and-release" strategy.

\subsection{Solid-phase synthesis of asymmetrical monomethine cyanines}

Tri-, penta- and heptamethine CDs can be yielded, as previously described, either by the "aldehyde" or the "hemicyanine" methods, while asymmetrical monomethine cyanines are synthetized by a slightly different approach, the "thioether method". This difference in synthetic procedure is even found in solid-phase methodology. Monomethine cyanines 1 and $\mathbf{2}$ were the first ever asymmetrical CDs synthetized by a solid-phase approach (Figure 8a) [37]. The dye 2, better known as Thiazole Orange (TO), has found large application in chemical biology to stain nucleic acids and proteins due to a remarkable fluorescence [46]. The first synthetic step in the preparation of $\mathbf{1}$ and $\mathbf{2}$ consisted on the attachment of either the picoline- 3 or the lepidine-based $\mathbf{4}$ precursors to the Rink amide MBHA polystyrene (PS) resin through the carboxylic function on the pendant chain (Figure $8 b)$. The quaternary salts $\mathbf{3}$ and $\mathbf{4}$ were coupled to the resin with a four-fold molar excess using the conventional reagents hexafluorophosphate benzotriazole tetramethyl uronium (HBTU) and $N, N$-diisopropylethylamine (DIEA) in
DMF/pyridine (1:1). The obtained coupled-resins intermediates 5 and $\mathbf{6}$ were subjected to react with the benzothiazole intermediate in four-fold molar excess in the presence of triethylamine (TEA) in dichloromethane (DCM) yielding the resin-coupled final product $\mathbf{7}$ and $\mathbf{8}$. Finally, the desired compounds $\mathbf{1}$ and $\mathbf{2}$ were cleaved from the resin by a treatment by a $95 \%$ trifluoroacetic acid aqueous solution. Monomethine cyanines $\mathbf{1}$ and $\mathbf{2}$ were obtained in almost quantitative yield and with high grade of purity through few simple solvent-rinsing [37].

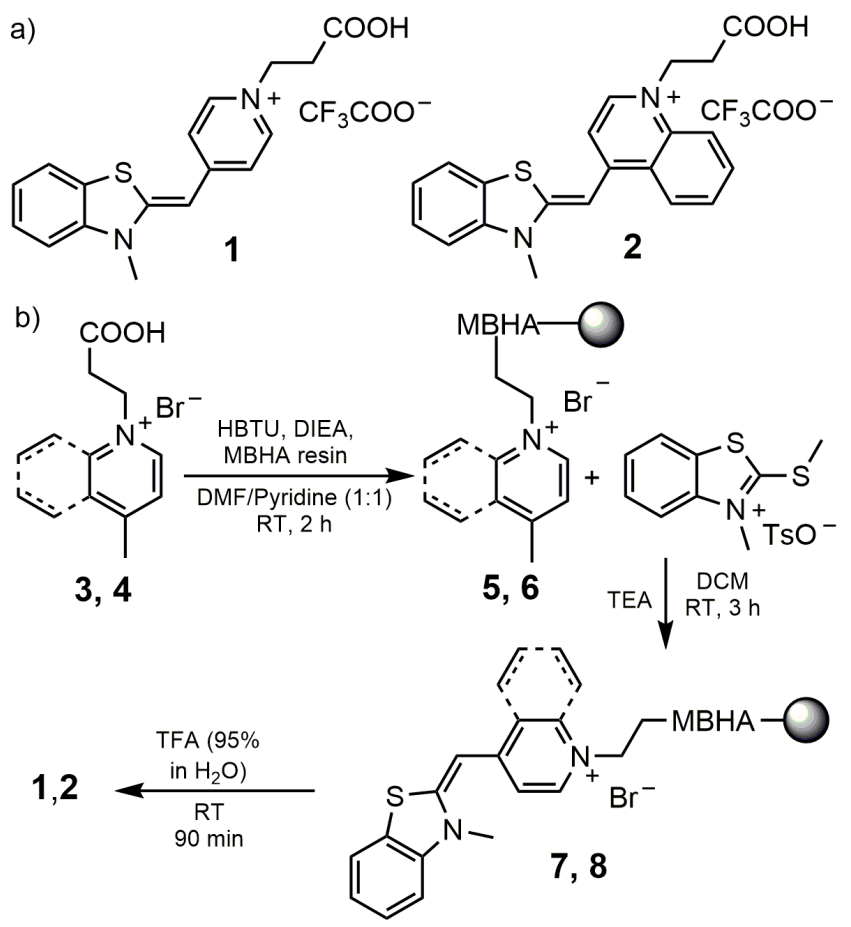

Figure 8. a) Asymmetrical monomethine 1 and $\mathbf{2}$ structures; b) Solid-phase synthesis of $\mathbf{1}$ and $\mathbf{2}$ with Rink amide MBHA PS resin.

Several other modified TO-based monomethine CDs 9-19 were synthetized by solid-phase method different from the one described for the preparation of compounds $\mathbf{1}$ and $\mathbf{2}$ (Figure 9a) [40]. While in the previous method, the resin was functionalized with a quaternary ammonium salts, the procedure to synthetize 9-19 involves: (i) the immobilization of a benzothiazole intermediate 20-24 onto Merrifield resin providing 25-29, (ii) the quaternarization of benzothiazole's nitrogen leading to the precursors $\mathbf{3 0 - 3 4}$ and finally (iii) the formation and later cleavage of the TO cyanine dyes from the resin (Figure 9b). Monomethine cyanines 9-19 were obtained with yield higher $90 \%$ with high grade of purity through few simple solvent-rinsing [40]. This approach closely resembles the above described "immobilized imidate" even if it does not involve directly an imidate intermediate. A particular attention has been dedicated to exploring the immobilization of the initial benzothiazoles 20-24 onto the Merrifield resin by evaluating the effect of different solvents, temperature, reaction time and substituents on the heterocycle. A good balance among those solvents' parameters have been shown to provide higher reaction conversion, while single high value of one of the solvent physical parameters is not enough to improve the reaction yield (Table 1). 
<smiles>[R1]CC[n+]1ccc(/C=C2\Sc3cc([R])c([R])cc3N2C)c2cccc(Cl)c21</smiles>

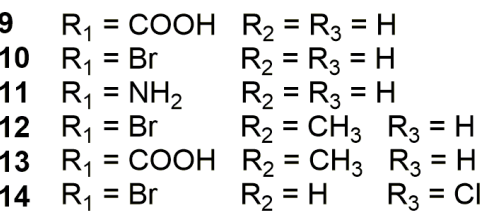



b)

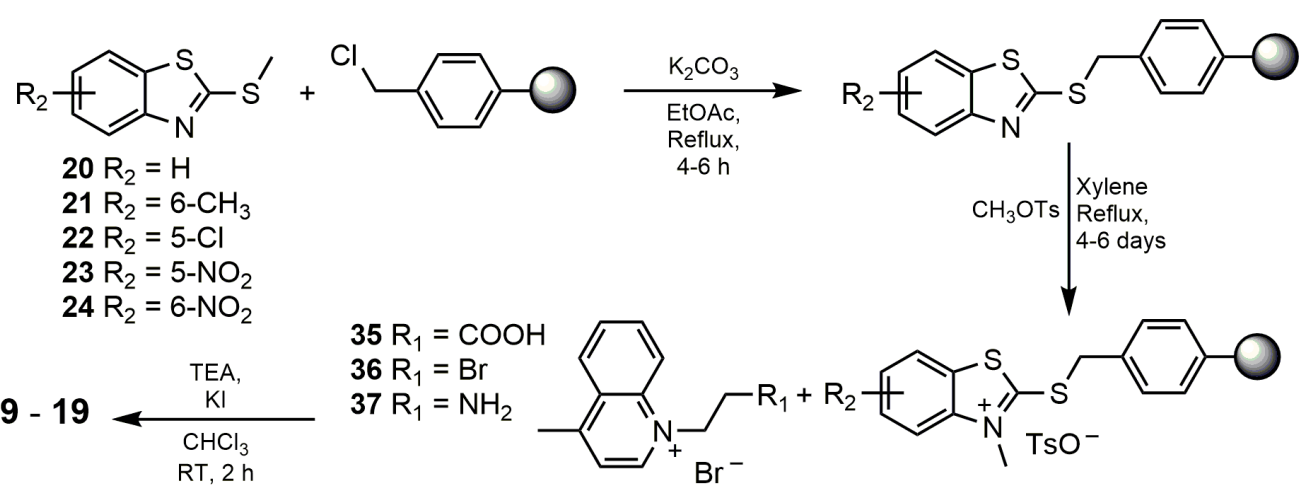

$25 \mathrm{R}_{2}=\mathrm{H}$

$26 \mathrm{R}_{2}=6-\mathrm{CH}_{3}$

$27 \mathrm{R}_{2}=5-\mathrm{Cl}$

$28 \mathrm{R}_{2}=5-\mathrm{NO}_{2}$

$29 \mathrm{R}_{2}=6-\mathrm{NO}_{2}$

Figure 9. a) Structure of the monomethine CDs 9-19 synthetized with Merrifield resin; b) Solid-phase synthesis of 9-19 with Merrifield resin by immobilization of benzothiazole derivatives, quaternarization of the immobilized-benzothiazole's nitrogen, formation and later cleavage of the TO cyanine dyes from the resin.

Ethyl acetate ensure the best conversion rate due to the good compromise among the above-mentioned parameters. Moreover, acetone and toluene have shown good results but the low boiling point of the former, and the low dielectric constant and dipole moment for the latter, negatively affect the conversion rate.

Table 1. Experimental conversion in different solvents

\begin{tabular}{ccccc}
\hline Solvent & $\begin{array}{c}\text { Boiling } \\
\text { point } \\
\left({ }^{\circ} \mathbf{C}\right)\end{array}$ & $\begin{array}{c}\text { Dielectric } \\
\text { constant } \\
\left(\mathbf{2 5}^{\circ} \mathbf{C}\right)\end{array}$ & $\begin{array}{c}\text { Dipole } \\
\text { moment } \\
(\mathbf{D})\end{array}$ & $\begin{array}{c}\text { Conversion } \\
\text { rate } \\
(\%))^{\boldsymbol{a}}\end{array}$ \\
\hline $\begin{array}{c}\text { Toluene } \\
\text { Ethyl }\end{array}$ & 110 & 2.40 & 1.33 & 85 \\
$\begin{array}{c}\text { acetate } \\
\text { Chloroform }\end{array}$ & 77 & 6.02 & 1.89 & 89 \\
Acetone & 56 & 4.81 & 1.15 & 76 \\
\hline
\end{tabular}

${ }^{a}$ Data acquired using 2-mercaptobenzothiazole. Reaction time: $4 \mathrm{~h}$.

Temperature and time also affect the overall reaction conversion in a remarkable way. Too low temperature has shown to hamper the product formation, while four hours has been found as sufficient time to maximize the product formation (Table 2). Finally, also the effect of the substituent on the benzothiazole ring has been investigated, suggesting that strong electron-withdrawing groups (e.g. $\mathrm{NO}_{2}$ ) can significantly lower the conversion rate without regards to the position on the benzothiazole scaffold.
Table 2. Reaction conversion screening

Temperature $\left({ }^{\circ} \mathrm{C}\right)$

\begin{tabular}{cccc} 
& $\mathbf{1 6}$ & $\mathbf{5 5}$ & reflux \\
\hline $\begin{array}{c}\text { Conversion } \\
\text { rate }(\%) \text { of } 20\end{array}$ & $10^{a}$ & $80^{a}$ & $89^{a}$ \\
\hline
\end{tabular}

Time (h)

\begin{tabular}{ccccc} 
& $\mathbf{1}$ & $\mathbf{2}$ & $\mathbf{4}$ & $\mathbf{6}$ \\
\hline $\begin{array}{c}\text { Conversion } \\
\text { rate (\%) of 20 }\end{array}$ & $43^{b}$ & $70^{b}$ & $89^{b}$ & $89^{b}$ \\
\hline
\end{tabular}

Substrate

\begin{tabular}{llllll} 
& $\mathbf{2 0}$ & $\mathbf{2 1}$ & $\mathbf{2 2}$ & $\mathbf{2 3}$ & $\mathbf{2 4}$ \\
\hline $\begin{array}{l}\text { Conversion } \\
\text { rate (\%) }\end{array}$ & $70^{b}$ & $66^{c}$ & $82^{c}$ & $38^{c}$ & $43^{c}$ \\
\hline$a$ & Reaction time: 4 h. Solvent: ethyl acetate. & & \\
$b$ & Solvent: ethyl acetate. Temperature: reflux. \\
${ }^{c}$ & Reaction time: 2 h Solvent: ethyl acetate. Temperature: reflux.
\end{tabular}

\subsection{Solid-phase synthesis of asymmetrical trimethine} cyanines

The asymmetrical trimethine CDs are the family of cyanines that has been most explored using solid-phase synthesis. Up to date just two asymmetrical trimethine cyanine, $\mathbf{3 8}$ and $\mathbf{3 9}$ have been synthetized with the method described for the monomethine scaffold [37]. Most of the reported CDs 40-53 by solid-phased synthesis were obtained either by the "catchand-release" method or the "immobilized imidate" method (Figure 10). 


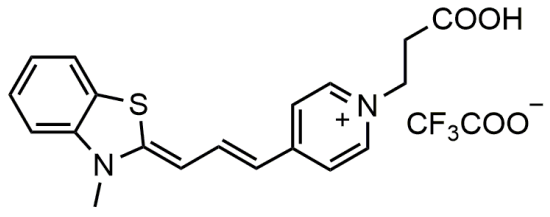

38

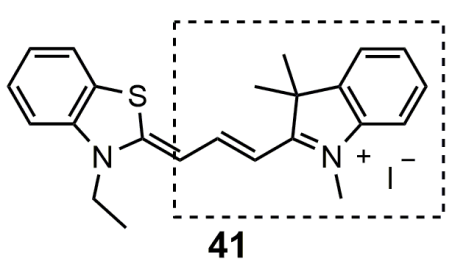

41


50



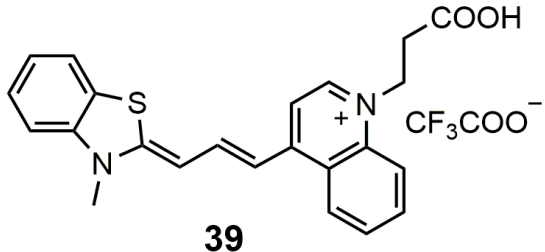


42
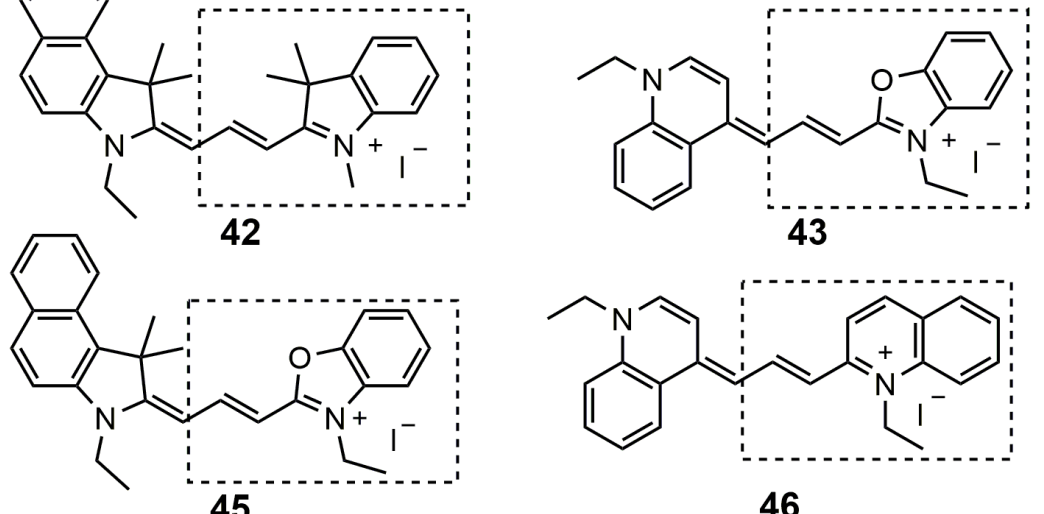

43



46

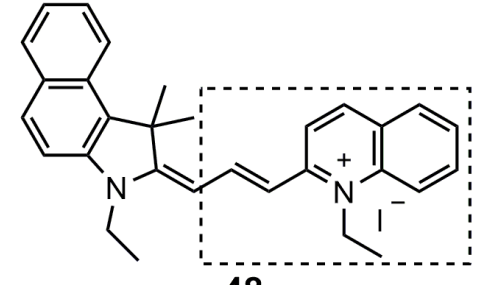

48<smiles>C/C=C1/Nc2ccc(S(=O)(=O)O)cc2C1(C)C</smiles>

HOOC

51


53

Figure 10. Asymmetrical trimethine cyanine dyes synthetized by solid-phase method; the moiety linked to the resin is indicated by the dashed box.

A systematic comparison of the solid-state synthesis using the two different methodology has been explored by varying both the resin-attached cyanine intermediate and the coupling ammonium quaternary salt yielding the dyes 40-48 (Table 3) [38, 39]. At first, the "catch and release" method has been explored by preparing three hemicyanine intermediates starting from the ammonium quaternary salts of an indole, a benzoxazole and a quinaldine scaffolds [38]. The purified hemicyanine were used in three-fold excess to reach with the sulfonyl chloride moieties on a resin in presence of DIEA, DCM at room temperature for four hours. Finally, the resin-attached hemicyanines were coupled with three different ammonium quaternary salts based on a benzoindole, a benzothiazole and a lepidine core respectively to provide the CDs 40, 41 and 43-47 with yields ranging from 19 to $79 \%$. The formation and cleavage reactions were performed using a 0.3 equivalents of quaternary ammonium salt, in binary solvent mixture of DIEA pyridine 1:9 for 30 minutes at room temperature. Interestingly, products $\mathbf{4 2}$ and 48 were not formed most likely due to the less reactivity of the benzoindole-based quaternary salt in comparison to the benzothiazole- and lepidine-based analogs. However, it was also reported that the low reactivity of benzoindole scaffold can be improved using a stronger base such as $\mathrm{NaH}$ [38]. Looking at the immobilized hemicyanine, the systematic investigation has highlighted that the indole-based core has led to higher product yields in comparison to the benzoxazole and quinaldine moieties while an immobilized benzoxazole scaffold has provided the higher values of compounds purity [38]. The "immobilized imidate" strategy, using the previously described combinations between the linked-intermediates and the quaternary ammonium salts, has generally provided CDs 40-48 in higher yields and better purities including, in this case, the cyanines 42 and 48 [39]. 

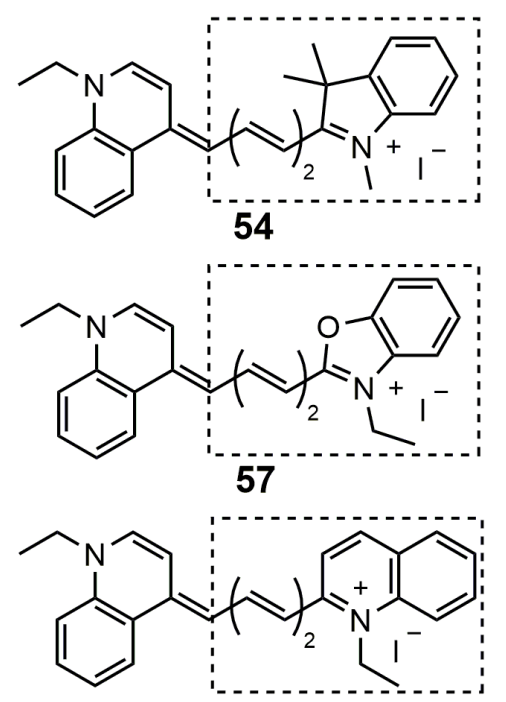

60



63
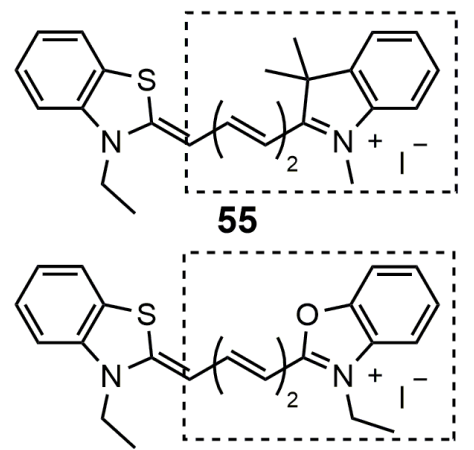

58

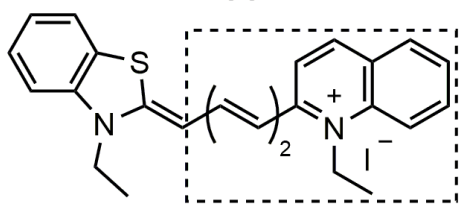

61

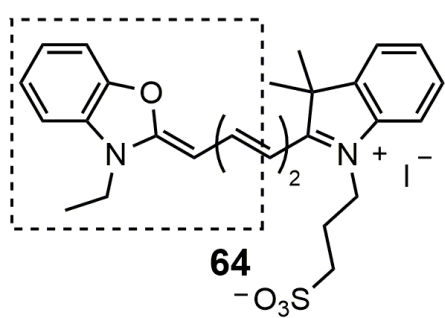



56

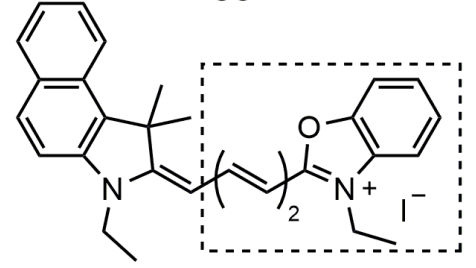

59



62

Figure 11. Asymmetrical pentamethine cyanine dyes synthetized by solid-phase method; the moiety linked to the resin is indicated by the dashed box.

Table 3. Reaction yield and product purity comparison

\begin{tabular}{|c|c|c|c|c|}
\hline \multirow[b]{2}{*}{ Cpd } & \multicolumn{2}{|c|}{ "Catch-and-release" } & \multicolumn{2}{|c|}{ "Immobilized imidate" } \\
\hline & Purity $^{a}$ & Yield $^{b}$ & Purity $^{a}$ & Yield $^{b}$ \\
\hline 40 & $89 \%$ & $79 \%$ & $>95 \%$ & $19 \%$ \\
\hline 41 & $91 \%$ & $60 \%$ & $>95 \%$ & $26 \%$ \\
\hline 42 & $<1 \%$ & - & $89 \%$ & $54 \%$ \\
\hline 43 & $>95 \%$ & $43 \%$ & $>95 \%$ & $58 \%$ \\
\hline 44 & $>95 \%$ & $23 \%$ & $>95 \%$ & $30 \%$ \\
\hline 45 & $50 \%$ & $32 \% c$ & $>95 \%$ & $76 \%$ \\
\hline 46 & $93 \%$ & $42 \%$ & $>95 \%$ & $70 \%$ \\
\hline 47 & $84 \%$ & $19 \%$ & $>95 \%$ & $23 \%$ \\
\hline 48 & $<1 \%$ & - & $50 \%$ & $53 \%$ \\
\hline
\end{tabular}

${ }^{a}$ Determined by HPLC with ELSD quantitation.

${ }^{b}$ Crude yield (based on amount of heterocycle in cleavage step).

${ }^{c}$ Improved yields and purities obtained by prior deprotonation with

$\mathrm{NaH}$ followed by reaction with immobilized hemicyanine.

In addition, water soluble trimethine CDs 49 and 50 were prepared with the same methodology with good yield of 73 and $59 \%$ respectively. It is worth nothing that the sulfonated heterocycles did not react well in the loading reaction with the imidate, while were providing good results as nucleophiles in the dye formation and cleavage step. Similar hydrophilic dyes, bearing sulfonic and/or carboxylic groups, based on an indole core 51, 52 and a benzoindole scaffold 53 have been prepared with the same methodology [41]. The overall reported yields of 94, 68 and $49 \%$ for $\mathbf{5 1 , 5 2}$, and $\mathbf{5 3}$ respectively have confirmed the lower reactivity of the benzoindole-based quaternary salts.

\subsection{Solid-phase synthesis of asymmetrical penta- and heptamethine cyanines}

The asymmetrical penta- and heptamethine cyanine dyes have been less explored by the solid-phase synthesis in comparison to the trimethine analogs. The "catch and release" method has been explored for the preparation of the pentamethine CDs but has shown limitation due to a competitive hydrolysis process [39]. On the other hand, the "immobilized imidate" approach has allowed the preparation of various dyes 54-64 in moderate to good yields (Figure 11) $[39,41]$.

Table 4. Reaction yield and product purity penta CDs

\begin{tabular}{cccccc}
\hline \multicolumn{5}{c}{ "Immobilized imidate" } \\
Cpd & Purity $^{a}$ & Yield $^{b}$ & Cpd & Purity $^{a}$ & Yield $^{b}$ \\
\hline $\mathbf{5 4}$ & $91 \%$ & $60 \%$ & $\mathbf{6 0}$ & $66 \% \%^{\mathrm{c}}$ & - \\
$\mathbf{5 5}$ & $>95 \%$ & $92 \%$ & $\mathbf{6 1}$ & $50 \%{ }^{\mathrm{c}}$ & - \\
$\mathbf{5 6}$ & $>95 \%$ & $96 \%$ & $\mathbf{6 2}$ & $76 \%$ & $57 \%$ \\
$\mathbf{5 7}$ & $>95 \%$ & $12 \%$ & $\mathbf{6 3}$ & $>95 \%$ & $64 \%$ \\
$\mathbf{5 8}$ & $>95 \%$ & $72 \%$ & $\mathbf{6 4}$ & $>95 \%$ & $86 \%$ \\
$\mathbf{5 9}$ & $92 \%$ & $46 \%$ & & & \\
\hline
\end{tabular}

${ }^{a}$ Determined by HPLC with ELSD quantitation.

${ }^{b}$ Crude yield (based on amount of heterocycle in cleavage step).

${ }^{c}$ Dyes not clearly separated by HPLC; purities estimated by ${ }^{1} \mathrm{H}-$ NMR integration.

The elongation of the polymethine linker itself has been shown to have unpredictable effect on the reaction yields for 54-64 when compared to the trimethine products (Table 4) [39]. For resin-linked substrate based on the indole core 5456 the yield in the final product is remarkably higher in comparison to the analogs $\mathbf{4 0 - 4 2}$ while the product purity is 
not much affected. Benzoxazole-based 57-59 were characterized by comparable degree of purity but lower product yield except for 58. Finally, the pentamethine CDs prepared by quinaldine-immobilized substrates 62-64 were affected by lower degree of purity for $\mathbf{6 2}$ and $\mathbf{6 3}$ which hampered the isolation of the desired dyes.

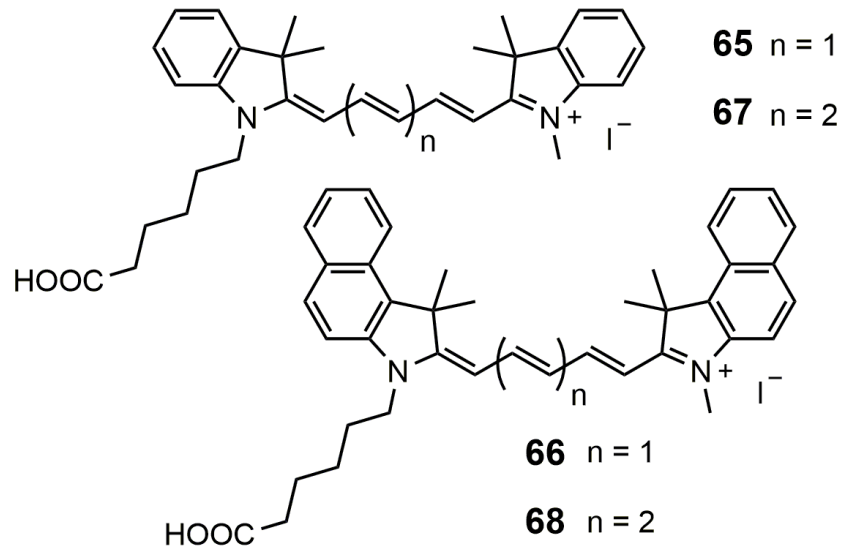

Figure 12. Asymmetrical penta- and heptamethine cyanine dyes synthetized by solid-phase method.

Extending the oligomethine bridge of $\mathbf{5 2}$ and $\mathbf{5 3}$ has provided the pentamethine CDs analogs $\mathbf{6 5}$ and $\mathbf{6 6}$ with a remarkable improve in the yields from 68 and $49 \%$ to 84 and $92 \%$ respectively (Figure 12) [41]. A further extension of the conjugated bridge allowed the solid-phase preparation of heptamethine derivatives $\mathbf{6 7}$ and $\mathbf{6 8}$ with a minimal to consistent drop in the product yields to 86 and $51 \%$ respectively.

\subsection{Water-soluble cyanine dyes using poly(ethylene glycol) as a soluble support}

The solid-phase synthesis of CDs has reduced the amount of chemical waste (e.g. solvent) needed for the nontrivial purifications required by the classical in solution protocols. Nevertheless, the above-described procedures in solid state still required the use of organic solvents to wet the resins and allow the reactions at the interface with the quaternary salts. An alternative approach using poly(ethylene glycol) (PEG) as a water-soluble support has been explored to synthesize unsymmetrical cyanines 69 and 70 analogs of the watersoluble 51 in an more environment friendly way (Figure 13) [46].

Even though this synthesis is not strictly conducted at the solid state, the comparison with the above strategies still provides useful information. The PEG resin is at first functionalized with para-aminobenzoic acid $\mathbf{7 1}$ to lead a free amine functionalized PEG $\mathbf{7 2}$ which is converted into $\mathbf{7 3}$ or 74 by reaction with the triethyl orthoformate or the 1,1,3,3tetramethoxypropane. Further reaction with the appropriate quaternarized nucleophile resulted in the formation of the dior tetra-methine bound hemicyanines $\mathbf{7 5}$ and $\mathbf{7 6}$. The final coupling with a second quaternary ammonium salts provided the desired asymmetrical CDs $\mathbf{7 7}$ and $\mathbf{7 8}$. The two dyes were isolated without the need of tricky purification in overall yields upon three steps of 22 and $24 \%$ respectively.

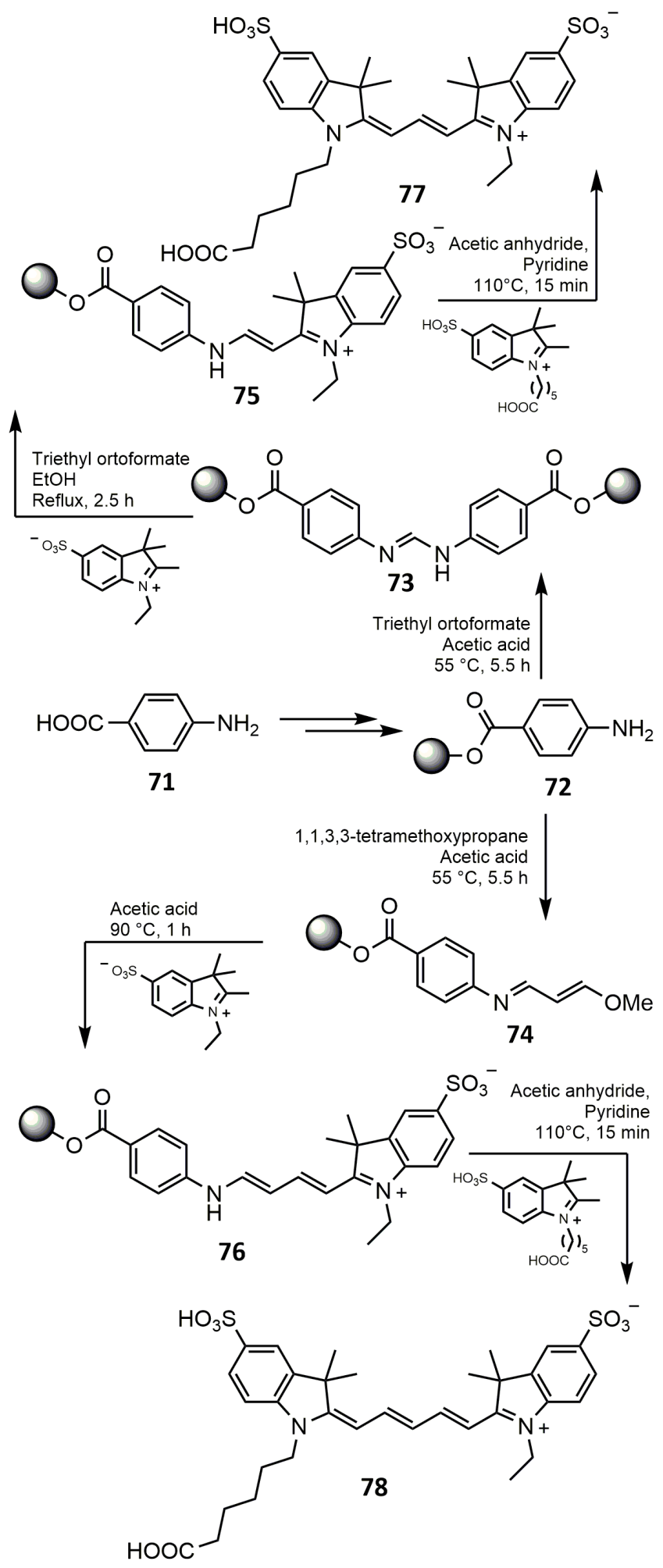

Figure 13. PEG-supported synthesis of trimethine 77 and pentamethine $\mathbf{7 8}$ cyanine dyes.

\section{SOLVENT-FREE SYNTHESIS OF ASYMMETRICAL CYANINES}

In the last two decades, alternative solid-state synthesis of cyanines have been explored even without the use of a resin support. Solvent-free reactions avoid the use of large amount of organic solvents, reducing the pollution and the costs [47]. The lack of the thermal energy required for the reaction, usually supplied by refluxing the reagent mixtures in 
solution, has been overcome by applying neat conditions, along with the microwave assisted heating, to achieve shorter reactions time, higher yields and easier purifications while lowering the formation of side products [48, 49]. Various hemicyanine 78-92 derivatives have been obtained by mixing an aromatic aldehyde 93-100 with the appropriate quaternary salt in presence of a catalytic amount of piperidine (Figure 14). The hemicyanine 78-84 were prepared withing two minutes proving yields between 8793\% for aldehyde bearing electron donating groups (EDG) $\mathbf{7 8 - 8 1}, 86 \%$ for the unsubstituted $\mathbf{8 2}$ and moderately lower yields of 92 and $93 \%$ for compounds 83 and 84 with decorated electron-withdrawing groups (EWG). A similar trend in the reaction yield, ranging from 73 to $99 \%$, was also depicted for the 4-linked hemicyanine series 85-92 over maximum reaction time up to five minutes with the reagent conversion improved and lowered by EDG and EWG respectively when compared to the unsubstituted product $\mathbf{8 9}$ [50].

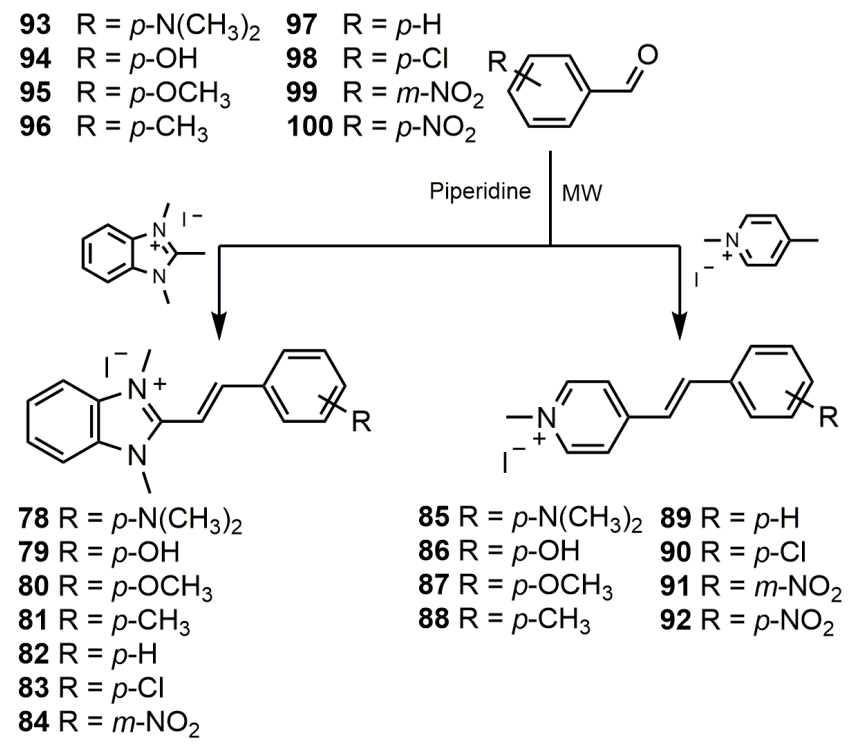

Figure 14. Hemicyanines 78-84 and 85-92 synthesized by microwave-assisted solvent-free synthesis.

A similar approach was described for the preparation of the monomethine cyanine scaffold of compound 101-104. Neither piperidine nor pyridine alone were able to catalyze the condensation reaction between the various quaternarized precursors 105-108 and the thioether substrate 109, most likely due to the strong and weak basicity respectively and for the rapid viscosity increase during the reaction (Figure 15a) [51]. The addition of a catalytic amount of triethylamine finally resulted in the formation of the cyanine 101-104 in high yields and short time. Dye 101, for example was obtained in $59 \%$ yield over fifteen minutes in comparison to the reported $21 \%$ over two hours by conventional method (Figure 15b) [52]. Moreover, it is of importance to highlight that carbonization of reactive materials and products can be simply limited by lowering fine setting the microwave power (Figure 15c).
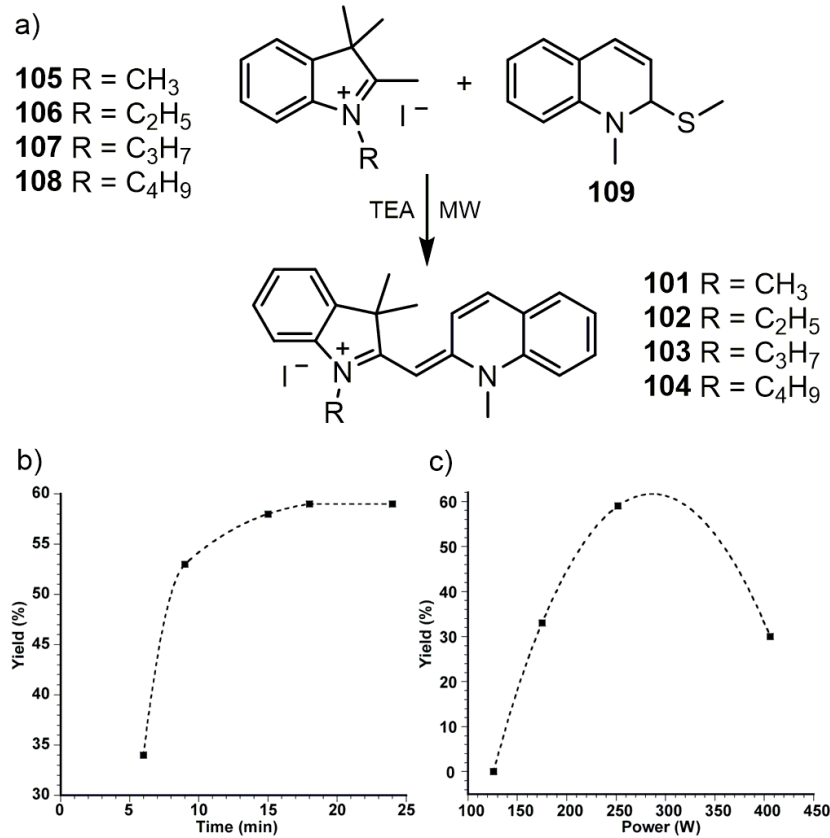

c)

Figure 15. a) Synthetic approach to the cyanines 101-104, b) the effect of time on yields, at a fixed power $(252 \mathrm{~W})$, for $\mathbf{1 0 1}, \mathrm{c})$ the effect of microwave power on yields of dye $\mathbf{1 0 1}$.

A final example of the neat condition synthesis of cyanine dye 110, whose perchlorate salt is widely used as biolabeling agent, was reported by mixing quaternary ammonium salt based on benzothiazole and the pyranone [53]. An initial grinding with a pestle, followed up but heating at $50^{\circ} \mathrm{C}$ for ten minutes, upon addition of a catalytic amount of perchloric acid in water afforded $\mathbf{1 1 0}$ in $30 \%$ yield while allowing the reusage of the unreacted materials (Figure 16).

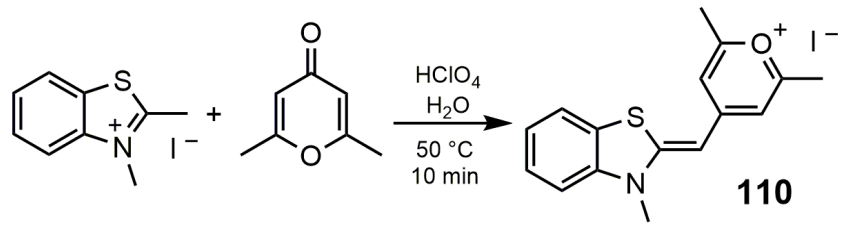

Figure 16. Synthesis of Cy39 iodide 110.

\section{CONCLUSION}

Cyanines history began more than a century ago and, over the years, have been tested and used in several research fields and applications. Nevertheless, the synthetic pathway and even more the purification protocols of this family of dyes have slowed down if not hampered their preparation and availability on the market. The synthesis of asymmetrical CDs and the isolation from the symmetrical side product is nowadays challenging for organic chemists. The solid-phase syntheses methodology has been explored in the preparation of the asymmetrical CDs, at first, on the most simple and small derivatives and lately allowing the preparation of water soluble heptamethine analogs. As a general consideration, these approaches allow synthesis with higher yield alongside higher purity rate of the final compound compared to the classical approaches. Additionally, the main advantage resides on the very easy purification procedures required to isolate the desired product from impurities with high structural similarity. It is easy to speculate that synthetic procedures which lower the 
consumption of organic solvents, the amount of waste while improving the product yields and reducing the reaction time will gain more and more importance in the next decades supported by the guideline of a more sustainable and environmental friendly economy and life.

\section{CONFLICT OF INTEREST}

The author(s) confirm that this article has no conflict of interest.

\section{ACKNOWLEDGMENTS}

This project has received funding from the European Union's Horizon 2020 research and innovation program under grant agreement no. 826013 (IMPRESSIVE). AF acknowledges the financial support from the Fondazione Cassa di Risparmio (CRT) di Torino, Italy (II tornata 2019 Project number: 2019.2260), CB and GR acknowledge the grant from Compagnia di San Paolo (Bando ExPost 2018).

\section{REFERENCES}

1. Hamer, F. M. (Ed.); The Cyanine Dyes and Related Compounds, Interscience Publishers, 1964.

2. Strekowski, L. (Ed.); Heterocyclic Polymethine Dyes, Springer, 2008.

3. Sturmer, D. M. Syntheses and Properties of Cyanine and Related Dyes. Chemistry of Heterocyclic Compounds 1977, 30, 441-587.

4. Mojzych, M.; Henary, M. Synthesis of Polymethine Dyes, Topics in Heterocyclic Chemistry, Springer, 2008, 14, 1-9.

5. Saccone, D.; Galliano, S.; Barbero, N.; Quagliotto, P.; Viscardi, G.; Barolo, C. Polymethine dyes in hybrid photovoltaics: structure-properties relationships. Eur. J. Org. Chem. 2016, 2244-2259.

6. West, W. First hundred years of spectral sensitization. Photographic Science and Engineering 1974, 18, 3548.

7. Sun, W.; Guo, S.; Hu, C.; Fan, J.; Peng, X. Recent development of chemosensors based on cyanine platforms. Chem. Rev. 2016, 116, 7768-7817.

8. Armitage, B. A. Cyanine dye-DNA interactions: intercalation, groove binding, and aggregation. Top. Curr. Chem. 2005, 253, 55-76.

9. Armitage, B. A. Cyanine dye-nucleic acid interactions. Top. Heterocycl. Chem. 2008, 14, 11-29.

10. Splitter, M. T.; Ehret, A.; Kietzmann, R.; Willig, F. Electron transfer threshold for spectral sensitization of silver halides by monomeric cyanine dyes. J. Phys. Chem. B 1997, 101, 2552-2557.

11. Levitus, M.; Ranjit, S. Cyanine dyes in biophysical research: the photophysics of polymethine fluorescent dyes in biomolecular environments. Q. Rev. Biophys. 2011, 44, 123-151.

12. Gorka, A. P.; Nani, R. R.; Schnermann, M. J. Harnessing cyanine reactivity for optical imaging and drug delivery. Acc. Chem. Res. 2018, 51, 3226-3235.

13. Pandey, R. K.; James, N.; Chen, Y.; Dobhal, M. P. Cyanine dye-based compounds for tumor imaging with and without photodynamic therapy. Top. Heterocycl. Chem. 2008, 14, 41-74.
14. Gorka, A. P.; Nani, R. R.; Schnermann. Cyanine polyene reactivity: scope and biomedical applications. Org. Biomol. Chem. 2015, 13, 7584-7598.

15. Shi, C.; Wu, J. B.; Pan, D.; Review on near-infrared heptamethine cyanine dyes as theranostic agents for tumor imaging, targeting, and photodynamic therapy. $J$. Biomed. Opt. 2016, 21, 50901.

16. Atchison, S.; Kamila, S.; Nesbitt, H.; Logan, K. A.; Nichola, D. M.; Fowley, C.; Davis, J.; Callan, B.; McHale, A. P.; Callan, J. F. Iodinated cyanine dyes: a new class of sensitizers for use in NIR activated photodynamic therapy (PDT). Chem. Comm. 2017, 53, 2009-2012.

17. Tian, H.; Meng, F. Solar cells based on cyanine and polymethine dyes. Optical Science and Engineering 2005, 99, 313-329.

18. Naim, W.; Novelli, V.; Nikolinakos, I.; Barbero, N.; Dzeba, I.; Grifoni, F.; Ren, Y.; Alnasser, T.; Velardo, A.; Borrelli, R.; Haacke, S.; Zakeeruddin, S. M.; Graetzel, M.; Barolo, C.; Sauvage, F. Transparent and Colorless Dye-Sensitized Solar Cells Exceeding 75\% Average Visible Transmittance JACSAu accepted DOI: https://dx.doi.org/10.1021/jacsau.1c00045

19. Ciubini, B.; Visentin, S.; Serpe, L.; Canaparo, R.; Fin, A.; Barbero, N. Design and synthesis of symmetrical pentamethine cyanine dyes as NIR photosensitizers for PDT. Dyes Pigments 2019, 160, 806-813.

20. Bertolino, C. A.; Ferrari, A. M.; Barolo, C.; Viscardi, G.; Caputo, G.; Coluccia, S. Solvent effect on indocyanine dyes: A computational approach Chem. Phys. 2006, 330, 52-59.

21. Bertolino, C. A; Caputo, G.; Barolo, C.; Viscardi, G.; Coluccia, S. Novel heptamethine cyanine dyes with large Stoke's shift for biological applications in the near infrared J. Fluoresc. 2006, 16, 221-5.

22. Benzi, C.; Bertolino, C. A.; Miletto, I.; Ponzio, P.; Barolo, C.; Viscardi, G.; Coluccia, S.; Caputo, G. The design, synthesis and characterization of a novel acceptor for real time polymerase chain reaction using both computational and experimental approaches Dyes Pigments 2009, 83, 111-120.

23. Alberto, G.; Miletto, I.; Viscardi, G.; Caputo, G.; Latterini, L.; Coluccia, S.; Martra, G. Hybrid CyanineSilica Nanoparticles: Homogeneous Photoemission Behavior of Entrapped Fluorophores and Consequent High Brightness Enhancement. J. Phys. Chem. C 2009, 113, 21048-21053.

24. Alberto, G.; Caputo, G.; Viscardi, G.; Coluccia, S.; Martra, G. Molecular Engineering of Hybrid Dye-Silica Fluorescent Nanoparticles: Influence of the Dye Structure on the Distribution of Fluorophores and Consequent Photoemission Brightness Chem. Mater. 2012, 24, 2792-2801.

25. Ilina, K.; Henary, M. Cyanine dyes containing quinoline moieties: history, synthesis, optical properties, and applications. Chem. Eur. J. 2021, 27, 4230-4248.

26. Demchenko, A. P. (Ed.); Advanced fluorescence reporters in chemistry and biology I, Springer, 2010. 
27. Mishra, A.; Behera, R. K.; Behera, P. K.; Mishra, B. K.; Behera, G. B. Cyanines during the 1990s: A Review. Chem. Rev. 2000, 100, 1973-2011.

28. Panigrahi, M.; Dash, S.; Patel, S.; Mishra, B. K. Synthesis of cyanines: a review. Tetrahedron 2012, 68, 781-805.

29. Henary, M.; Levitz, A. Synthesis and applications of unsymmetrical carbocyanine dyes. Dyes Pigments 2013, 99, 1107-1116.

30. Owens, E. A.; Bruschi, N.; Tawney, J. G.; Henary, M. A microwave-assisted and environmentally benign approach to the synthesis of near-infrared fluorescent pentamethine cyanine dyes. Dyes Pigments 2015, 113, 27-37.

31. Merrifield, R. B. Solid phase peptide synthesis. I. The synthesis of a tetrapeptide. J. Am. Chem. Soc. 1963, 85, 2149-2154.

32. El-Faham, A.; Albericio, F. Peptide coupling reagents, More than a letter soup. Chem. Rev. 2011, 111, 65576602.

33. Li, Q.; Lee, J.-S.; Ha, C.; Park, C. B.; Yang, G.; Gan, W. B.; Chang, Y.-T. Solid-phase synthesis of styryl dyes and their application as amyloid sensors. Angew. Chem. Int. Ed. 2004, 43, 6331-6335.

34. Er, J. C.; Tang, M. K.; Chia, C. G.; Liew, H.; Vendrell, M.; Chang, Y.-T. Fluorescent dye cocktail for multiplex drug-site mapping on human serum albumin. Chem. Sci. 2013, 4, 2168-2176.

35. Vendrell, M.; Krishna, G. G.; Ghosh, K. K.; Zhai, D.; Lee, J.-S.; Zhu, Q.; Yau, Y. H.; Shochat, S. G.; Kim, H.; Chung, J.; Chang, Y.-T. Solid-phase synthesis of BODIPY dyes and development of an immunoglobulin fluorescent sensor. Chem. Commun. 2011, 47, 84248426.

36. Kopecky, K.; Novakova, V.; Miletin, M.; Kucera, R.; Zimcik, P. Solid-phase synthesis of azaphthalocyanineoligonucleotide conjugates and their evaluation as new dark quenchers of fluorescence. Bioconjugate Chem. 2010, 21, 1872-1879.

37. Isacsson, J.; Westman, G. Solid-phase synthesis of asymmetric cyanine dyes. Tetrahedron Lett. 2001, 42, 3207-3210.

38. Mason, S. J.; Balasubramanian, S. Solid-phase catch, activate, and release synthesis of cyanine dyes. Org. Lett. 2002, 4, 4261-4264.

39. Mason, S. J.; Hake, J. L.; Nairne, J.; Cummins, W. J.; Balasubramanian, S. Solid-phase methods for the synthesis of cyanine dyes. J. Org. Chem. 2005, 70, 2939-2949.

40. Fei, X.; Yang, S.; Zhang, B.; Liu, Z.; Gu, Y. Solidphase synthesis and modification of Thiazole Orange and its derivatives and their spectral properties. $J$. Comb. Chem. 2007, 9, 943-950.

41. Lopalco, M.; Koini, E. N.; Cho, J. K.; Bradley, M. Catch and release microwave mediated synthesis of cyanine dyes. Org. Biomol. Chem. 2009, 7, 856-859.

42. Das, R. K.; Samanta, A.; Ha, H.-H.; Chang, Y.-T. Solid phase synthesis of ultra-photostable cyanine NIR dye library. RSC Advances 2011, 1, 573-575.

43. Brooker, L. G. S.; White, F. L.; Keyes, G. H.; Smyth, E. P.; Oesper, P. F. Color and constitution. II.1 Absorptions of some related vinylene-homologous series. J. Am. Chem. Soc. 1941, 63, 3192-3203.

44. Z. Peng, H. J. Geise, X. Zhou, B. Peng, R. Carleer, R. Dommisse. The structure of benzimidazole cyanine dyes, their spectroscopy, and their performance in photographic emulsions. Liebigs Ann./Recl. 1997, $27-$ 33.

45. Okamoto, A. Thiazole Orange-tethered nucleic acids and ECHO Probes for fluorometric detection of nucleic acids, Topics in Nucleic Acids and Molecular Biology, Springer, 2016, 63-81.

46. Jiang, L.-L.; Dou, L.-F.; Li, B.-L. An efficient approach to the synthesis of water-soluble cyanine dyes using poly(ethylene glycol) as a soluble support. Tetrahedron Lett. 2007, 48, 5825-5829.

47. Tanaka, K.; Toda, F. Solvent-free organic synthesis. Chem Rev., 2000, 100, 1025-1074.

48. De la Hoz, A.; Diaz-Ortiz, A.; Moreno, A. Microwaves in organic synthesis. Thermal and non-thermal microwave effects. Chem. Soc. Rev., 2005, 34, 164 178.

49. Chen, P.; Rosana, M. R.; Dudley, G. B.; Stiegman, A. E. Parameters affecting the microwave-specific acceleration of a chemical reaction. J. Org. Chem. 2014, 79, 7425-7436.

50. Wang, L.; Zhang, X.; Shi, Y.; Zhang, Z. Microwaveassisted solvent-free synthesis of some hemicyanine dyes. Dyes Pigments, 2004, 62, 21-25.

51. Fu, Y.-L.; Huang, W.; Li, C.-L.; Wang, L. Y.; Wei, Y.S.; Huang, Y.; Zhang, X.-H.; Wen, Z.-Y.; Zhang, X.-Z. Monomethine cyanine dyes with an indole nucleus: Microwave-assisted solvent-free synthesis, spectral properties and theoretical studies. Dyes Pigments, 2009, 82, 409-415.

52. Ficken, G. E.; Kendall, J. D. The reactivity of the alkylthio-group in nitrogen ring compounds. Part II: cyanine bases from 3,3-dimethyl-2-methylthio-3Hindole. J. Am. Chem. Soc. 1960, 1529-1536.

53. Sereda, G. A. Organic solvent-free synthesis of Cy39 iodide. Tetrahedron Lett. 2009, 50, 973-974. 